\title{
A Rare Case of Three Distinct Gastrointestinal Neoplasms Occurring Simultaneously in an Elderly Patient
}

\author{
Saad Hanan ${ }^{\mathrm{a}, \mathrm{f}} @$, Enkhmaa Luvsannyam $^{\mathrm{b}}$, Molly S. Jain ${ }^{\mathrm{a}}$, Srishti Laller ${ }^{\mathrm{c}}$, Tayyab Cheema ${ }^{\mathrm{d}}$, \\ Cory Mellon ${ }^{\mathrm{d}}$, Don Teed ${ }^{\mathrm{d}}$, Manoj Somagutta ${ }^{\mathrm{e}}$, Laila N. Ahmad ${ }^{\mathrm{a}}$, \\ Frederick M. Tiesenga ${ }^{d}$
}

\begin{abstract}
Duodenal adenocarcinoma (DA), gastrointestinal stromal tumor (GIST), and pancreatic neuroendocrine tumor (PNET) are a set of rare gastrointestinal (GI) cancers characterized by nonspecific symptoms such as anemia, weight loss, and abdominal pain. We report an interesting case of DA, GIST, and PNET in a 79-year-old African American male who presented to the emergency department with syncope. The patient was tachycardic and found to have severe anemia. Further computed tomography (CT) of the abdomen and pelvis with contrast revealed a mass within the duodenal bulb along with a pancreatic cystic lesion. The patient underwent gastroduodenectomy and distal pancreatectomy with incidental findings of GIST in the posterior gastric wall. The patient after surgical removal of all tumors no longer experienced abdominal pain and had hematocrit and hemoglobin $(\mathrm{H} \& \mathrm{H})$ level of 9.1/31.7 postoperation day (POD) 14 . The case unfolds a rare presentation of three neoplasms that were moderate to well-differentiated with no metastases. It highlights the importance of assessment of rare GI neoplasms concurrently with colorectal cancer when geriatric patients present with nonspecific GI symptoms.
\end{abstract}

Keywords: Neoplasm; Gastrointestinal; Anemia; Duodenal adenocarcinoma; Gastrointestinal stromal tumor; Pancreatic neuroendocrine tumor

\section{Introduction}

Gastrointestinal (GI) cancers are becoming more prevalent

Manuscript submitted August 18, 2021, accepted September 1, 2021

Published online September 29, 2021

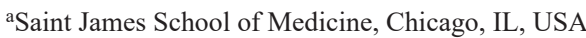

${ }^{b}$ Avalon University School of Medicine, Chicago, IL, USA

${ }^{\mathrm{c}}$ Caribbean Medical University, Rosemont, IL, USA

${ }^{\mathrm{d}}$ West Suburban Medical Centre, Oak Park, IL, USA

${ }^{\mathrm{e} C a l i f o r n i a}$ Institute of Behavioral and Neurosciences and Psychology, Fairfield, CA, USA

${ }^{f}$ Corresponding Author: Saad Hanan, Saint James School of Medicine, Chicago, IL, USA. Email: Saad_uni@hotmail.com

doi: https://doi.org/10.14740/jmc3765 with an estimated rise of 4 million new cases each year worldwide including the USA $[1,2]$. Among the GI cancers, colorectal cancer, gastric cancer, pancreatic cancer, and liver cancer are the most common types while neuroendocrine tumors, gastrointestinal stromal tumors (GISTs), and anal cancer are less common $[1,2]$. Various risk factors are associated with GI cancers such as cigarette smoking, alcohol consumption, diets high in saturated fats, gastroesophageal reflux disease, Helicobacter pylori infection, and inflammatory bowel disease [3]. Symptoms of GI cancer depend on the specific organ getting affected and mostly show up during the advanced stages of cancer. Common diagnostic tests include endoscopy, colonoscopy, biopsy, imaging studies such as magnetic resonance imaging (MRI), X-ray, ultrasound, and computed tomography (CT) scan or positron emission tomography (PET) scan $[1,2]$. Treatment options consist of chemotherapy, radiation therapy, surgical resection, or palliative care when found at an advanced or unresectable stage $[1,2]$.

Duodenal adenocarcinoma (DA) represents a rare group of GI cancers with only $1 \%$ prevalence, although it is one of the most aggressive types of GI cancer [4]. Along with an unhealthy diet, duodenal polyps, duodenal adenomas, and Gardner syndrome are also risk factors for DA [4]. It typically presents with nonspecific symptoms such as abdominal pain, nausea, fatigue, vomiting, and weight loss. With progression, symptoms such as anemia, GI obstruction, and jaundice may also be evident [4]. Pancreaticoduodenectomy or segmental duodenal resections are the common treatment modalities for earlier stages of cancer when metastasis has not started [5]. For patients with higher rates of recurrence, adjuvant chemotherapy and radiation therapy should be considered [5]. The prognostic factors with the worst outcomes include the patient's age, lymph node metastasis, and high-grade tumor [4].

GISTs are the common mesenchymal tumors comprising about $0.1-3 \%$ of GI malignancies [6]. The pathophysiology for these benign tumors underlies the mutation of tyrosine kinase genes that lead to the malignant expression of the cells involving the lineage of interstitial cells of Cajal [7]. The three main types of GISTs are spindle cell type (70\%), epithelioid cell type (20\%), and mixed type (10\%) [5]. More than $95 \%$ of GISTs stain positive for KIT protein (CD117), and approximately $85 \%$ carry a mutation in the $c-K I T$ gene or plateletderived growth factor receptor alpha (PDGFRA) gene [8]. Patients with GIST also present with nonspecific symptoms such 


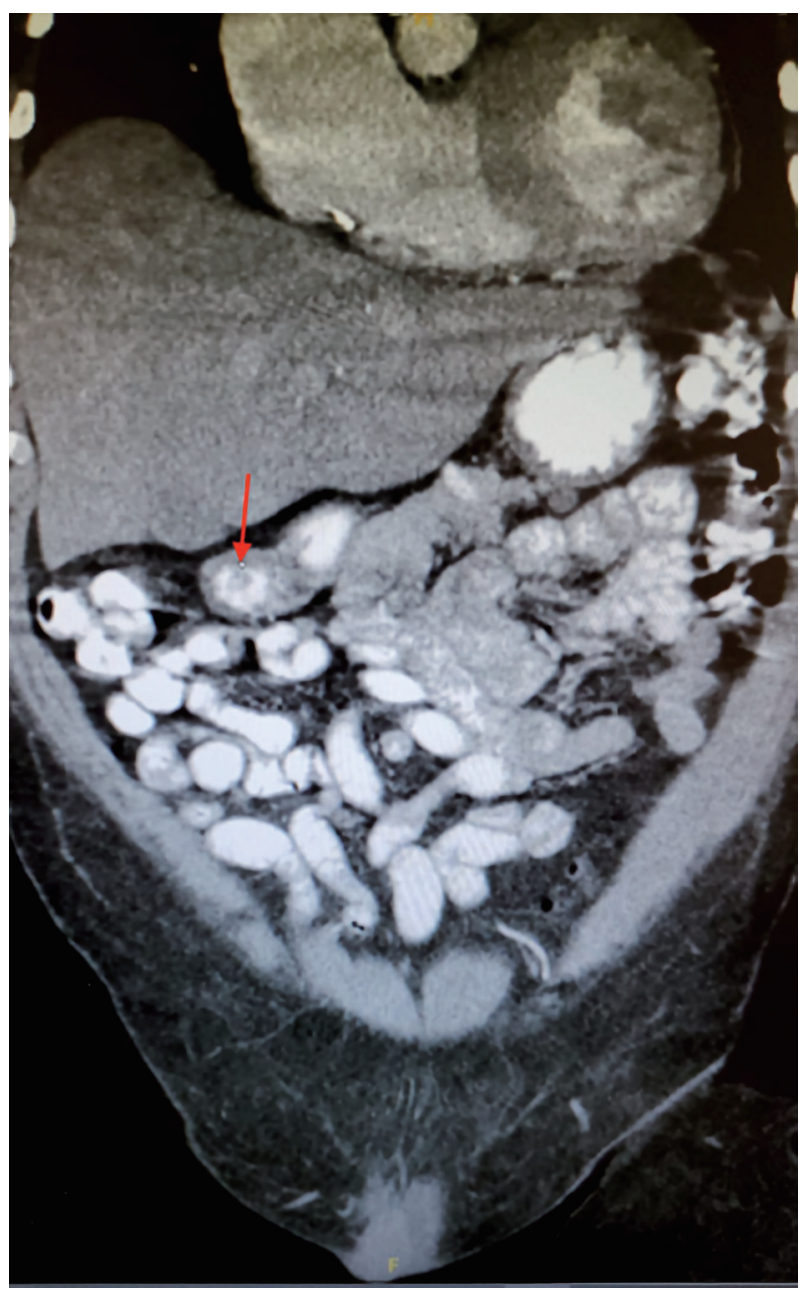

Figure 1. The CT scan in coronal view of the abdomen showcasing 1.3-cm duodenal filling defect (red arrow). CT: computed tomography.

as abdominal pain and GI bleeding either as hematochezia or anemia [8]. This tumor is usually found incidentally, and surgical resection is the mainstay of treatment [7]. Also, tyrosine kinase inhibitors such as imatinib could be used for metastatic or unresectable tumor cases [6].

Pancreatic neuroendocrine tumors (PNETs) are neuroendocrine in origin expressing neural phenotypes [9]. These tumors are distinguished based on the functioning of hormones or nonfunctioning [9]. The functioning PNETs consist of insulinoma, glucagonoma, gastrinoma, and VIPoma while the non-functioning PNETs cause mortality through tissue invasion [9]. About $10 \%$ PNETs are also associated with familial endocrine tumor syndromes as multiple endocrine neoplasia type 1 (MEN1), von Hippel-Lindau disease (VHL), neurofibromatosis type 1 (NF1), and tuberous sclerosis (TSC) [10]. Measurement of neuroendocrine tumor markers such as neuron-specific enolase (NSE), chromogranin (CGA), and pancreastatin is used for diagnostic confirmation and treatment follow-up [10]. The management of PNETs focuses on surgery, locoregional therapy, systemic therapy, and complication control [10].

We describe a unique case of the three aforementioned GI

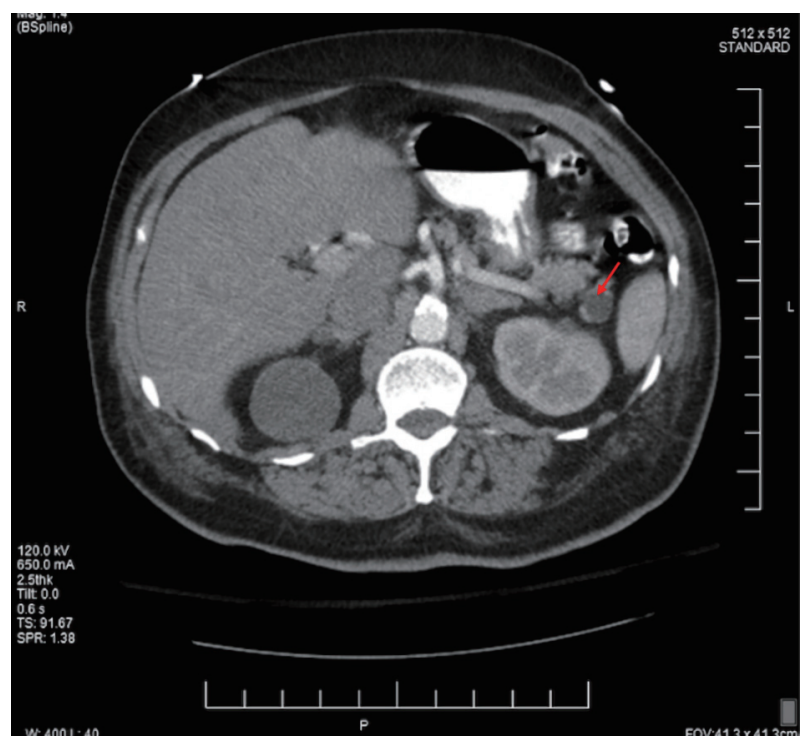

Figure 2. The CT scan in axial view of the abdomen showcasing 1.5$\mathrm{cm}$ pancreatic cyst (red arrow). CT: computed tomography.

cancers presenting simultaneously in an elderly patient with symptomatic anemia and other clinical implications.

\section{Case Report}

\section{Investigations}

We present a case of a 79-year-old African American male with a past medical history of diabetes mellitus type II, essential hypertension, and atrial fibrillation with symptomatic normocytic anemia resulting from three distinct GI cancers. The patient was treated with gastroduodenectomy with Billroth II anastomosis, gastric fundus resection, and distal pancreatectomy. The patient initially presented to the emergency department (ED) with syncope and tachycardia. He reported experiencing symptoms of fatigue, abdominal pain, and lightheadedness for 2 months. Complete blood count revealed a hemoglobin level of $4.9 \mathrm{~g} / \mathrm{dL}$ requiring three packed red blood cell transfusions.

\section{Diagnosis}

On the following day, due to his symptoms and concurrent severe anemia, the GI mass was suspected, and a CT of the abdomen and pelvis with contrast was promptly ordered. The findings of the CT report demonstrated a $1.3-\mathrm{cm}$ ovoid filling defect in duodenal bulb (Fig. 1) and a 1.5-cm cystic lesion in the pancreatic tail (Fig. 2). Furthermore, the patient completed an esophagogastroduodenoscopy (EGD) which exhibited a mass in the duodenal bulb that was partially resected for biopsy. The biopsy confirmed invasive adenocarcinoma of gastroduodenal origin with a histological grade of III/III under World Health Organization (WHO) classification. 

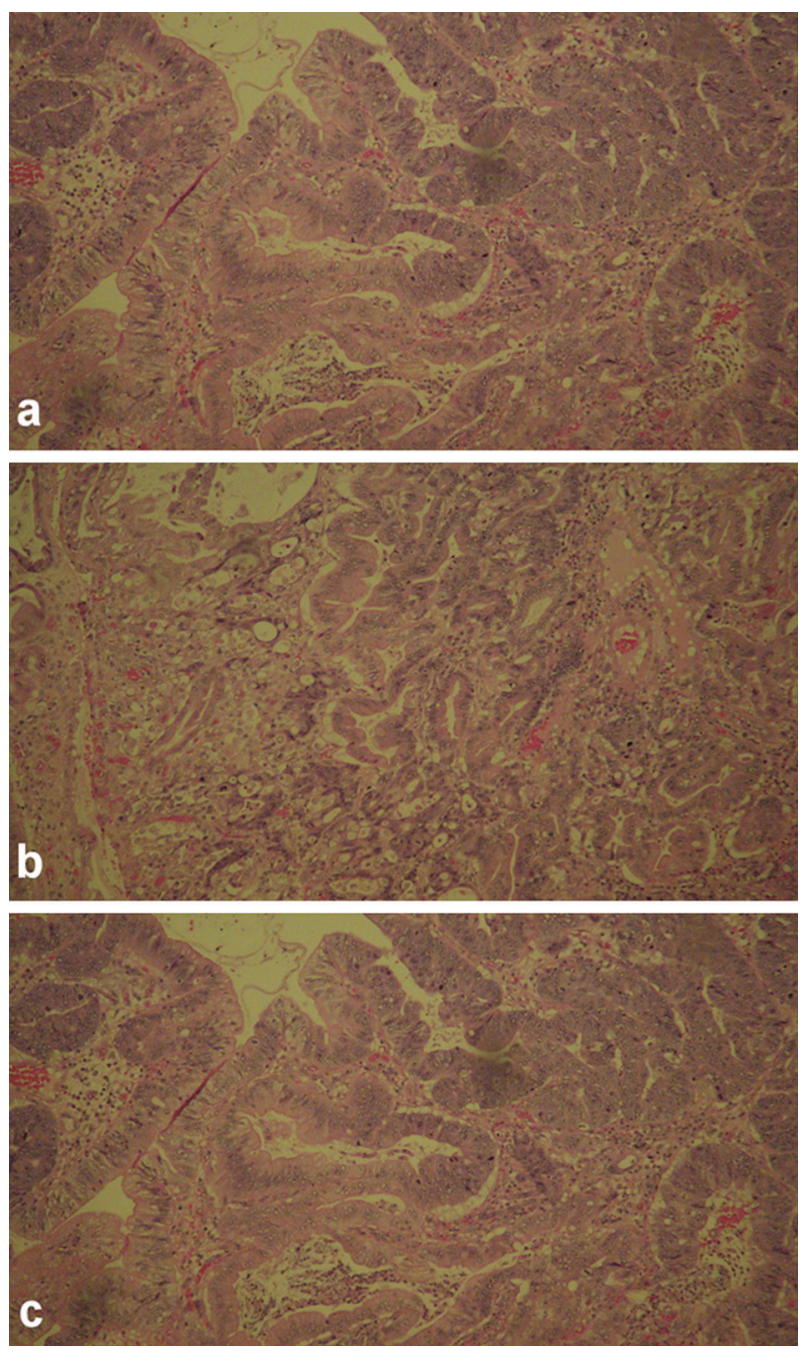

Figure 3. The histology images of in situ gastroduodenal adenocarcinoma, infiltrating into submucosa.

\section{Treatment}

The patient underwent exploratory laparoscopic resection of the duodenal bulb mass and pancreatic tail mass. During the surgical removal of the duodenal bulb tumor, the examination of the stomach showcased a pedunculated mass near the fundus on the posterior wall of the stomach. The posterior wall mass was excised with duodenal and pancreatic masses and all samples were sent to pathology.

The pathological report for proximal duodenum mass showed an in situ and invasive well to moderately differentiated adenocarcinoma measuring $4.5 \times 2.3 \times 2.3 \mathrm{~cm}$ with infiltration into the submucosa (Fig. 3). The posterior gastric wall mass was reported to be a GIST, $1.3 \times 1.1 \times 0.8 \mathrm{~cm}$ in size. This tumor was of low grade, spindle cell type, with dystrophic microcalcifications (Fig. 4). The cells stained positive with Dog1, CD34, and c-KIT. The mass on the tail of the pancreas was shown as a well differentiated neuroendocrine tumor measuring $0.8 \times 0.5 \times 0.5 \mathrm{~cm}$. The tumor stained positive for

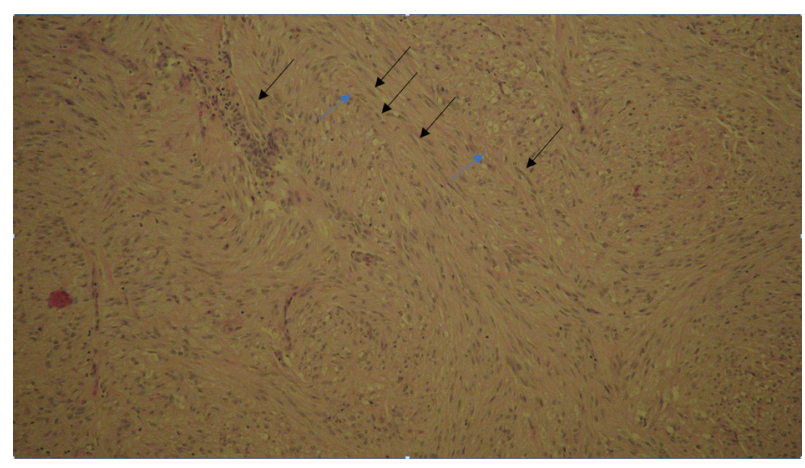

Figure 4. Gastrointestinal stromal tumor, low grade, spindle cell type with dystrophic (black arrow) microcalcifications (blue arrow).

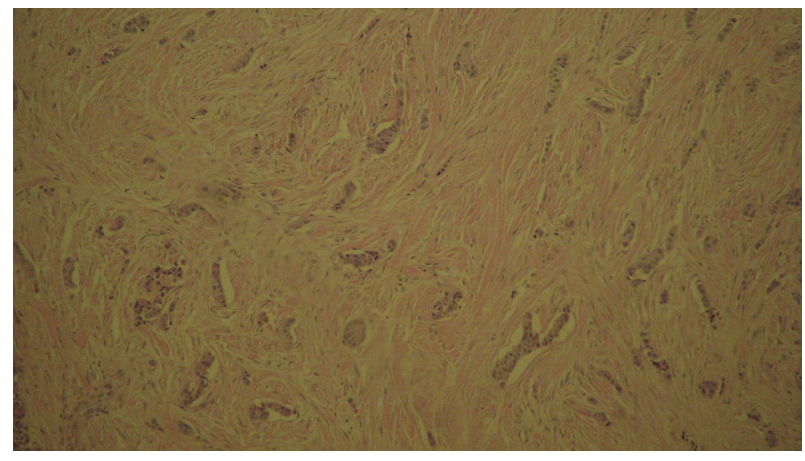

Figure 5. Pancreatic intraepithelial, well differentiated neuroendocrine tumor history slide stained with synaptophysin.

AE1/AE3, CAM5.2, and diffusely strong staining with neuroendocrine markers (synaptophysin, chromogranin (CGA), neuron-specific enolase (NSE), and CD56) (Fig. 5). The oncology report demonstrated that all of the cancers were excised completely with clean margins and therefore, no chemotherapy treatment was planned.

\section{Follow-up and outcomes}

The patient's postoperative course revolved around management of any complications related to surgery and maintaining the hemoglobin levels. The patient's postoperation day (POD) 1 hemoglobin/hematocrit $(\mathrm{H} \& \mathrm{H})$ levels were 10.2/35.1. The H\&H levels on POD 7 were 8.9/29.8 and POD 14 were $9.1 / 31.7$. The patient did not report further abdominal pain and was no longer having episodes of syncope. The patient is currently recovering well.

\section{Discussion}

DAs are usually diagnosed at an advanced stage due to atypical symptoms or resemblance for duodenal ulcer [11]. In our case, the tumor was found at an early stage most likely due to the concurrent two other tumors. The DA did not involve the head of the pancreas; thus, the proximal duodenal resection 
was only performed. Due to the rare incidence of duodenal cancer, there is a lack of evidence in the current literature. The screening protocol, treatment approach, and clinical prognostic factors are still controversial [11]. Currently, the best management includes CT scan for screening, EGD for biopsy, and surgery for tumor removal [12]. Further studies are necessary to investigate the control of the disease and if adjuvant chemotherapy or radiotherapy can improve patient survival rate.

GISTs occur most commonly in the stomach $(60-70 \%)$, and least common in the colorectum $(10 \%)$ and esophagus $(<1 \%)$ [13]. The majority of GISTs are asymptomatic which can lead to misdiagnosis such as leiomyomas, sarcomas, and schwannomas, and delayed diagnosis [8]. The number of patients with metastasis has increased significantly due to delayed diagnoses [8]. In our case, the GIST was also found incidentally during the patient's exploratory laparotomy to remove the duodenal and pancreatic masses. A multidisciplinary approach with the correct characterization of the tumor is required for disease management. Mutational analysis is also required as the tumor is known to be resistant to traditional treatments. Surgery is the only curative treatment for resectable tumors [8]. Our patient had a non-metastatic disease and was treated by gastric fundic wedge resection with no further therapy.

The diagnosis of PNETs is often delayed due to the complex presentation of hormonal hypersecretion syndromes and nonspecific symptoms of non-functional PNETs [10]. Imaging, endocrine testing, histological and biochemical testing are required to accurately diagnose PNETs [9]. Liver metastasis is the most common cause of death in patients with untreated PNETs [9]. A biopsy is critical in grading the tumor and identifying hormones and markers which are necessary to confirm pancreatic origin in case of liver metastases [9]. In our case, the patient's pancreatic lesion was found on CT and biopsy confirmed a non-functioning, low-grade PNET that was positive for CGA, synaptophysin, NSE, and CD56. Along with surgical removal, somatostatin analogs such as octreotide are useful for functioning PNETs and chemotherapy is beneficial for high-grade PNETs [9]. A study showed that tyrosine kinase inhibitors such as sunitinib prolonged survival in patients with well differentiated PNETs, but it has not been studied in depth [9].

The highlight of this case is the presentation of all three distinct neoplasms in the GI system simultaneously. All three tumors, DA, GIST, and PNET, have different characteristics and may present with various clinical manifestations depending on their location and stage. While DA and GIST mainly occur in 60 - 70 years of age, PNET can present in younger patients with a family history of familial endocrine tumor syndromes. Our patient does not have a family history of cancers and is not tested positive for any gene mutations involved in GIST and PNET making it quite unique for him to be affected by these malignancies. Physicians should suspect concurrent bleeding mass when a patient presents with severe symptomatic anemia and an early-stage tumor. Moreover, physicians should consider other GI cancers in their differential diagnoses instead of suspecting the traditional diagnosis of colorectal cancer in an old patient with anemia. The consequence of coincident neoplasms can cause extensive GI bleeding and lead to life-threatening syncope. Because all three tumors may present with nonspecific symptoms, early evaluation plays an important role in timely diagnosis and treatment.

\section{Conclusions}

Colorectal cancer is the most common cause of anemia in the USA, especially in the geriatric population. However, this case presents less common GI cancers as DA, GIST, and PNET to be the cause of symptomatic anemia. We present a rare case of these three distinct GI cancers of independent origin within the abdomen, concurrently in an older patient. Because these tumors present with nonspecific symptoms, it can be challenging to evaluate and diagnose them early. Hence, it is prudent for clinicians to consider rare GI tumors in the differential diagnoses for geriatric populations presenting with nonspecific GI symptoms. It is also important for primary care physicians to follow up with their geriatric patients with regular physical examinations and appropriate management interventions in a timely manner.

\section{Acknowledgments}

We thank Dr. Robert Wlodarski, Department of Radiology, and West Suburban Medical Centre for their contributions in this case report.

\section{Financial Disclosure}

None to declare.

\section{Conflict of Interest}

The abstract of this case study has been published as a poster presentation at the following link: https://www.cureus.com/ posters/1822-a-case-showcasing-three-rare-gastrointestinalcancers-in-an-anemic-patient.

\section{Informed Consent}

We obtained written informed consent from the patient for publication of this case report and accompanying images.

\section{Author Contributions}

Saad Hanan was in charge of writing the case and structure/ flow of the paper. Enkmaa Luvsannyam was in charge of writing the discussion. Molly S. Jain was in charge of writing the introduction. Srishti Laller was in charge of writing the abstract/conclusion. Tayyab Cheema was in charge of leading the editing team. Cory Mellon, Don Teed and Manoj Somagutta were part of the editing team. Laila N. Ahmad was part of writing the introduction/discussion and editor. Frederick M. 
Tiesenga was the head surgeon for the case and the individual behind the information provided in the case.

\section{Data Availability}

The authors declare that data supporting the findings of this study are available within the article.

\section{Abbreviations}

DA: duodenal adenocarcinoma; GIST: gastrointestinal stromal tumor; PNET: pancreatic neuroendocrine tumor; GI: gastrointestinal; CT: computed tomography; EGD: esophagogastroduodenoscopy; CGA: chromogranin; NSE: neuron-specific enolase; H\&H: hemoglobin/hematocrit; POD: post operation day

\section{References}

1. Bray F, Ferlay J, Soerjomataram I, Siegel RL, Torre LA, Jemal A. Global cancer statistics 2018: GLOBOCAN estimates of incidence and mortality worldwide for 36 cancers in 185 countries. CA Cancer J Clin. 2018;68(6):394424.

2. Sung H, Ferlay J, Siegel RL, Laversanne M, Soerjomataram I, Jemal A, Bray F. Global cancer statistics 2020: GLOBOCAN Estimates of incidence and mortality worldwide for 36 cancers in 185 countries. CA Cancer J Clin. 2021;71(3):209-249.

3. Correa P. Gastric cancer: overview. Gastroenterol Clin North Am. 2013;42(2):211-217.
4. Cloyd JM, George E, Visser BC. Duodenal adenocarcinoma: Advances in diagnosis and surgical management. World J Gastrointest Surg. 2016;8(3):212-221.

5. Meijer LL, Alberga AJ, de Bakker JK, van der Vliet HJ, Le Large TYS, van Grieken NCT, de Vries R, et al. Outcomes and treatment options for duodenal adenocarcinoma: a systematic review and meta-analysis. Ann Surg Oncol. 2018;25(9):2681-2692.

6. Burch J, Ahmad I. Gastrointestinal Stromal Cancer. In: StatPearls. Treasure Island (FL), 2021.

7. Zhao X, Yue C. Gastrointestinal stromal tumor. J Gastrointest Oncol. 2012;3(3):189-208.

8. Sanchez-Hidalgo JM, Duran-Martinez M, Molero-Payan R, Rufian-Pena S, Arjona-Sanchez A, Casado-Adam A, Cosano-Alvarez A, et al. Gastrointestinal stromal tumors: A multidisciplinary challenge. World J Gastroenterol. 2018;24(18):1925-1941.

9. Ro C, Chai W, Yu VE, Yu R. Pancreatic neuroendocrine tumors: biology, diagnosis, and treatment. Chin J Cancer. 2013;32(6):312-324.

10. Sun J. Pancreatic neuroendocrine tumors. Intractable Rare Dis Res. 2017;6(1):21-28.

11. Kim MJ, Choi SB, Han HJ, Park PJ, Kim WB, Song TJ, Suh SO, et al. Clinicopathological analysis and survival outcome of duodenal adenocarcinoma. Kaohsiung J Med Sci. 2014;30(5):254-259.

12. Wang Z, Ding Z, Huang S, Zhong S. Experience in clinical diagnosis and treatment of duodenal tumors. Mol Clin Oncol. 2016;5(6):731-739.

13. Liu Z, Zheng G, Liu J, Liu S, Xu G, Wang Q, Guo M, et al. Clinicopathological features, surgical strategy and prognosis of duodenal gastrointestinal stromal tumors: a series of 300 patients. BMC Cancer. 2018;18(1):563. 